

\title{
Analysis of the influence of inertia for non-contact micromanipulation
}

Mohamed Dkhil, Aude Bolopion, Stéphane Regnier, Michaël Gauthier

\section{To cite this version:}

Mohamed Dkhil, Aude Bolopion, Stéphane Regnier, Michaël Gauthier. Analysis of the influence of inertia for non-contact micromanipulation. Journal of Micro-Bio Robotics, 2017, 13, pp.1 - 12. hal02963675

\section{HAL Id: hal-02963675 \\ https://hal.science/hal-02963675}

Submitted on 11 Oct 2020

HAL is a multi-disciplinary open access archive for the deposit and dissemination of scientific research documents, whether they are published or not. The documents may come from teaching and research institutions in France or abroad, or from public or private research centers.
L'archive ouverte pluridisciplinaire HAL, est destinée au dépôt et à la diffusion de documents scientifiques de niveau recherche, publiés ou non, émanant des établissements d'enseignement et de recherche français ou étrangers, des laboratoires publics ou privés. 


\title{
Analysis of the influence of inertia for non-contact micromanipulation
}

\author{
Mohamed Dkhil • Aude Bolopion • \\ Stéphane Régnier • Michaël Gauthier
}

Received: date / Accepted: date

This article aims at analyzing the effect of the inertia of the objects in remotely actuated systems at the micrometer scale. As the size decreases inertia is commonly neglected and the systems are considered quasi-static. However, this article shows that for high velocities (around $8 \mathrm{~mm} / \mathrm{s}$ ) the dynamic behavior of the manipulated particle must be taken into account. To perform this analysis, a remotely magnetically actuated system dedicated to high speed manipulation is used. $60 \mu \mathrm{m}$-size particles placed at the air/liquid interface are actuated in $2 \mathrm{D}$ at different velocities. Precise trajectory tracking is obtained for velocities up to $2.8 \mathrm{~mm} / \mathrm{s}$ (around 50 body lengths per second), for which inertia can be neglected. For faster velocities (more than 140 body lengths per second demonstrated in this paper) phase lag appears in trajectory tracking: inertia needs to be considered for the control. Experimental results are corroborated by numerical analysis of the model of the system. This article paves the way toward the control of future high speed remotely actuated systems at the micrometer scale.

Mohamed Dkhil

Institut FEMTO-ST, Univ. Bourgogne Franche-Comté, CNRS, 24 rue Alain Savary, 25000 Besançon, France.

Institut des Systèmes Intelligents et de Robotique, Université Pierre et Marie Curie, CNRS UMR 7222, 4 Place Jussieu, 75005 Paris, France.

Aude Bolopion and Michaël Gauthier

Institut FEMTO-ST, Univ. Bourgogne Franche-Comté, CNRS, 24 rue Alain Savary, 25000 Besançon, France.

E-mail: aude.bolopion@femto-st.fr,michael.gauthier@femto-st.fr

Stéphane Régnier

Institut des Systèmes Intelligents et de Robotique, Université Pierre et Marie Curie, CNRS UMR 7222, 4 Place Jussieu, 75005 Paris, France.

E-mail: regnier@isir.upmc.fr 
Keywords Inertia - Non contact micromanipulation - 2D trajectory control · Magnetic actuation · Air/liquid interface

\section{Introduction}

To handle artificial components or biological cells of size less than $100 \mu \mathrm{m}$, "no weight robots", which are based on the remote application of forces, have been proposed [1]. This technique allows to avoid the inertia of the manipulator to reach high throughput and to avoid direct contact with the object to avoid adhesion issues.

This non-contact manipulation has elicited widespread interest over the past few years [2]. Various physical principles can be considered. Among the most common ones are laser trapping [3], dielectrophoresis [4], [5], acoustophoresis [6], [7] and magnetophoresis [8], [9], [10]. However, most of the work is carried out inside liquids [11], [12], [13], which limits the velocity due to the drag force. Ambient environments have been investigated but the adhesion between the objects and the substrate makes repeatability of actioning challenging [14].

An other approach consists in using the interface between two immiscible fluids [15], [16], [17]. In particular, the air/liquid interface benefits from the low adhesion of the liquid, and the low viscous drag of the air [18]. The weight of micrometer size particles is lower than surface tension, so that the objects lay on the surface.

In the previously mentioned systems, as the size decreases, the inertia of the manipulated objects is commonly neglected. For a given applied force the objects experience huge accelerations. As a consequence the permanent regime is attained almost instantaneously: these systems are supposed to be quasistatic. [19], [1], [20], [21]. Until now, as the velocity of the objects remained low enough this approximation hold. However, to guarantee the interest of non contact manipulation for future micro assembly systems, or future highly selective cell sorting, one of the key challenge is to increase the velocity of the manipulation. We show in this article that the limit at which inertia must be taken into account can be reached with micromanipulation systems dedicated to high speed. To do so, we have presented in our previous work [18] magnetic actuation at the air/liquid interface. The low drag force in this environment, as well as the compact magnetic actuation system allows for high speed manipulation. In this paper we use this setup to analyze closed loop control of particles of $60 \mu \mathrm{m}$. Since it is technically not possible to vary the mass of the object while keeping the same magnetic properties and drag coefficient this paper proposes to analyze the trajectory of the object for different inputs. At low speed the system can be considered as quasi static and precise trajectory tracking is achieved. The commonly admitted approximation which consists in neglecting inertia is verified. However as the speed increases the dynamic behavior of the object is visible. Phase lag in the trajectory tracking appears, which is typical of inertia. Numerical simulation is performed, based on the model developed in [22], to corroborate this hypothesis and to analyze the 
influence of inertia. The analysis provided in this paper will be of utmost importance for the control of the future high speed micromanipulation systems.

This paper is organized as follows. Section 2 presents the model of a magnetically actuated micromanipulation system at the air/liquid interface. In Sec. 3 experiments are performed on a $60 \mu \mathrm{m}$ particle for different velocities to analyze the influence of inertia. Numerical analysis is proposed in Sec. 4 to corroborate the analysis. Sec. 5 concludes the paper.

\section{Magnetic actuation at the air/liquid interface}

\subsection{Basic equations}

A micrometer size particle placed at the air/liquid interface in a magnetic actuation platform undergoes four forces: (i) the magnetic force $\mathbf{F}_{m}$ induced by the coils, (ii) the weight of the particle $\mathbf{P}$, (ii) the force resulting from the surface tension $\mathbf{T}$ and (iv) the drag force $\mathbf{F}_{d}$, as shown in Fig. 1. Based on Newton's second law, the movement of the particle can be written as:

$$
m \mathbf{a}=\mathbf{P}+\mathbf{F}_{m}+\mathbf{F}_{d}+\mathbf{T}
$$

where a and $m$ are respectively the acceleration and the mass of the particle. This basic equation describes the dynamic behavior of the particle as a function of the magnetic force.

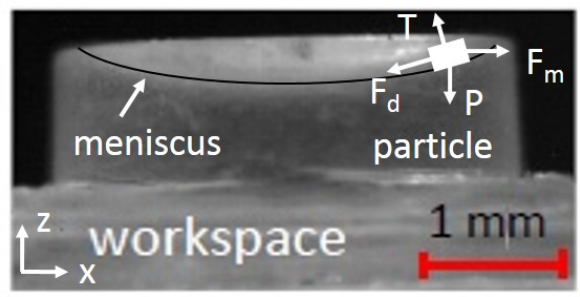

Fig. 1 Forces acting on a magnetically actuated micrometer size particle placed at the air/liquid interface of a millimeter size reservoir. Due to the small size of the reservoir a concave meniscus can be seen at the interface. $\mathbf{F}_{m}$ is the magnetic force induced by the coils, $\mathbf{P}$ is the weight of the particle, $\mathbf{T}$ is the force resulting from the surface tension and $\mathbf{F}_{d}$ is the drag force. The picture depicts the side view of the reservoir. A sketch of the particle is drawn on the picture (drawing not to scale).

\subsection{Experimental setup}

The experimental setup is composed of a magnetic actuation platform and the environment on which a micrometer size particle is placed. The magnetic actuation platform is composed of four orthogonal coils actuated by four currents 
$I_{1}, I_{2}, I_{3}$ and $I_{4}$ as described in Figure 2. They are powered by an electronic amplifier (ST TL048CN) which amplifies the control signals. The magnetic field produced by the platform is the sum of the magnetic field produced by each coil. Since the four coils are identical the magnetic field is measured and identified by a polynomial function for one of the coil. Rotation and translation are applied to this function to get the magnetic field produced by the three other coils. The magnetic field is measured on coil number 3 in this paper. It can be decomposed into two components, $B_{x}$ and $B_{r}$, which are the magnetic fields along the axial (resp. radial) direction of the coil. The radial magnetic field is neglected in this paper.

The axial magnetic field is measured using a Hall-effect sensor. To measure the magnetic field at different distances from the coil, the coil is mounted on a motorized micromanipulator so that its position can be precisely controlled. The distance between the coil and the Hall sensor varies from 0 to $10 \mathrm{~mm}$ with a step of $100 \mu \mathrm{m}$. Fig. 3 presents the experimentally measured magnetic field $B_{x} . B_{x}$ is approximated by a polynomial function $f(x)$ which represents the ratio between the axial magnetic field $B_{x}(r=0, x)$ produced by the coil and the current $I_{3}$ which is used to power the coil:

$$
B_{x}(r=0, x)=f(x) \cdot I_{3}
$$

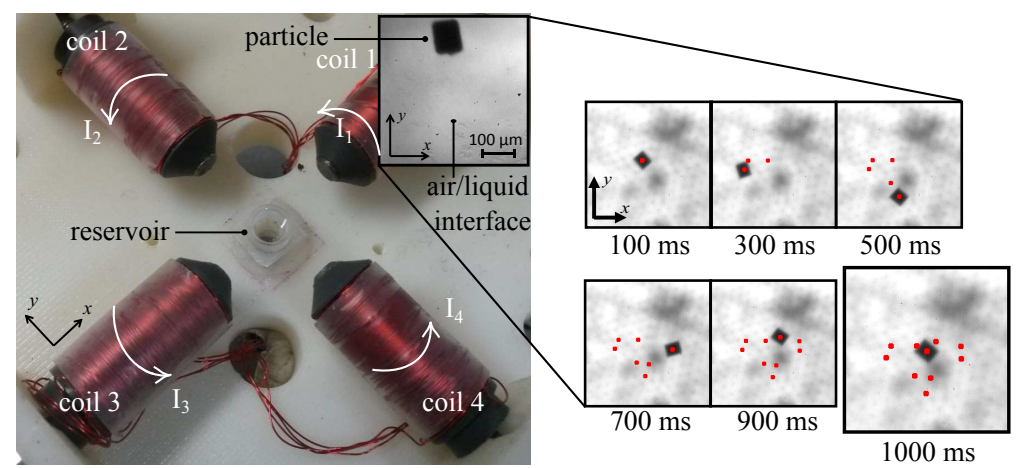

Fig. 2 Experimental setup for non contact magnetic actuation at the air/liquid interface. On the right, an example of trajectory tracking is depicted. A $60 \times 50 \times 25 \mu \mathrm{m}^{3}$ particle is controlled to perform a "heart-like" trajectory in $T=1 \mathrm{~s}$.

During the experiments performed in the next section (Fig. 6) the maximum absolute value of the current set to the coils is around $1.2 \mathrm{~A}$. The particle is situated at around $100 \mu \mathrm{m}$ from the center of the reservoir $(x= \pm 100 \mu \mathrm{m})$. The axial magnetic field produced by one coil at this position is around $20 \mathrm{mT}$ (see inset of Fig. 3). The radial magnetic field is neglected since the particle is situated at around $\pm 100 \mu \mathrm{m}$ from the axis of the coil (coil diameter: around $1 \mathrm{~cm})$. 


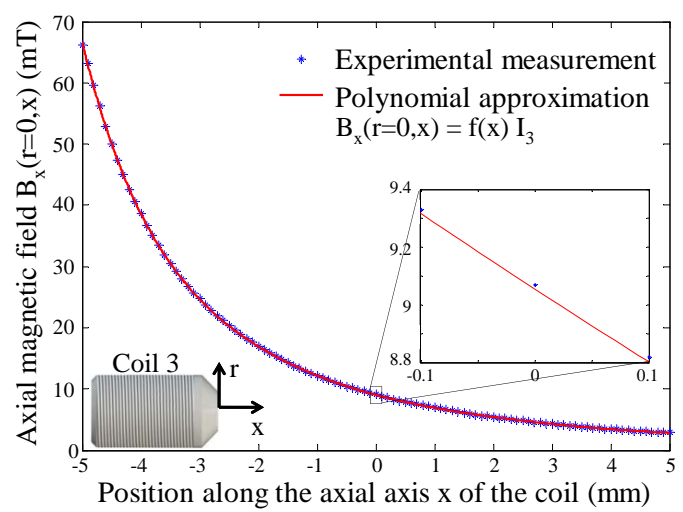

Fig. 3 Axial magnetic field produced by coil 3 when powered by a current $I_{3}=0.5 \mathrm{~A}$. It can be approximated using a polynomial function $f(x)$ which represents the ratio between the axial magnetic field $B_{x}(r=0, x)$ of coil 3 powered by the current $I_{3}$. The coil is situated at $5 \mathrm{~mm}$ from the center of the reservoir $(x=-5 \mathrm{~mm})$. The position $x=0$ corresponds to the center of the reservoir. The inset represents the magnetic field in the workspace (i.e. in the center of the reservoir).

The environment is a $4 \mathrm{~mm}$-diameter cylindrical reservoir filled with distilled water (density: $1000 \mathrm{~kg} . \mathrm{m}^{-3}$, dynamic viscosity: 0.001 Pa.s, surface tension: $\left.72.8 \cdot 10^{-} 3 \mathrm{~N} . \mathrm{m}^{-1}\right)$. The center of the reservoir is situated $5 \mathrm{~mm}$ from the coils. A micrometer size particle is placed at the air/liquid interface. This particle is made of nickel electrodeposited in a clean room. The thickness of the nickel layer is around $25 \mu \mathrm{m}$. The overall dimensions of the particle are $60 \times 50 \times 25 \mu^{3}$. This setup is placed on an antivibration table (Newport SG Breadboard).

The control unit is composed of a PC running software on a real time Unix Kernel, and of a camera. The software is written in $\mathrm{C}$ using OpenCV libraries for image processing. It controls the signals sent to the coils via a National instrument card (NI-PCI-6733). A "Photonfocus" camera mounted on Mitutoyo M plan Apo 5X objective records images of the workspace (part of the environment which contains the particle). The sampling time of the camera is set to $2 \mathrm{~ms}$. The images are imported to a computer using a PCI interface.

A detailed description of the experimental setup can be found in [18]. This setup has proved to be able to generate high speed trajectories since it is directly inspired from MagPier, the microrobotic system which has won the NIST Microrobotics Challenge which consists in performing a given trajectory in the shortest time [23]. To improve repeatability, so that particles of $60 \mu \mathrm{m}$ can be manipulated, the environment has been modified to use an air/liquid interface. 


\subsection{Dynamic Modeling}

The basic equation (1) has been used to build a dynamic modeling of the particle movement. Readers interested in the details of the calculation and the complete expression of the forces acting on the particle should refer to our previous work [22]. A summary is provided in this section since the model is used for closed loop control (Sec. 3) and numerical simulation (Sec. 4).

The device has four inputs, the four coils currents, which can be concretely reduced to two independent control variables $I_{x}$ and $I_{y}$ verifying:

$$
\begin{array}{lll}
\text { if } I_{x} \geq 0, & I_{1}=I_{x}, & I_{3}=0 \\
\text { if } I_{x} \leq 0, & I_{1}=0, & I_{3}=-I_{x} \\
\text { if } I_{y} \geq 0, & I_{2}=I_{y}, & I_{4}=0 \\
\text { if } I_{y} \leq 0, & I_{2}=0, & I_{4}=-I_{y}
\end{array}
$$

Considering (i) a perfect geometry of the system, (ii) that the coils are similar and (iii) that the magnetization of the ferromagnetic particle does not reach saturation (see Sec. 2.4), we have shown that the magnetic forces are expressed by [22]:

$$
\left\{\begin{array}{l}
F_{m x}=V \chi\left(2 f(x) f^{\prime}(x) \operatorname{sign}\left(I_{x}\right) I_{x}^{2}+f^{\prime}(y) f(x) I_{x} I_{y}\right) \\
F_{m y}=V \chi\left(2 f(y) f^{\prime}(y) \operatorname{sign}\left(I_{y}\right) I_{y}^{2}-f^{\prime}(x) f(y) I_{x} I_{y}\right)
\end{array},\right.
$$

in which $F_{m x}$ (resp. $F_{m y}$ ) is the magnetic force along the $x$ (resp. $y$ ) axis, $V$ is the volume of particle, $\chi$ is its magnetic susceptibility and $f^{\prime}$ is the derivative of the function $f$ defined in Sec. 2.2.

From the equation (1), we also have shown that the dynamic behavior $M_{d y n}$ of the particle is given by:

$$
M_{d y n}:\left\{\begin{array}{l}
m \ddot{x}+k \dot{x}+\left(\frac{m g}{R_{c}}\right) x=F_{m x} \\
m \ddot{y}+k \dot{y}+\left(\frac{m g}{R_{c}}\right) y=F_{m y}
\end{array},\right.
$$

in which $m$ is the mass of the particle, $k$ is the Stockes coefficient of the drag force. $R_{c}$ is the radius of curvature of the meniscus present at the air/liquid interface due to the small size of the reservoir (some millimeters of diameter). It is assumed that the particle does not deform the interface significantly as discussed in [18] and in Sec. 4.2. This model is the most generic one and will be used to simulate the device behavior. It takes into account the inertia of the particle.

A second model $M_{k i n}$ is used to build the control laws. This simplified model considers that both axis $x$ and $y$ are decoupled and that the inertia of the particle can be neglected. This hypothesis about inertia is commonly made at this scale [19], [1], [20], [21]. However its validity is questionable and 
the limit of this model will be discussed. From (7) and (8), the kinetic model verifies:

$$
M_{k i n}:\left\{\begin{array}{l}
k \dot{x}+\left(\frac{m g}{R_{c}}\right) x=2 V \chi f(0) f^{\prime}(0) \operatorname{sign}\left(I_{x}\right) I_{x}^{2} \\
k \dot{y}+\left(\frac{m g}{R_{c}}\right) y=2 V \chi f(0) f^{\prime}(0) \operatorname{sign}\left(I_{y}\right) I_{y}^{2}
\end{array} .\right.
$$

\subsection{Particle's characteristics}

The model presented above necessitates to know several characteristics of the particle, such as its mass $m$, its volume $V$, its magnetic susceptibility $\chi$ and its drag coefficient $k$.

The volume is obtained from the geometric dimensions of the particle (60x50x $25 \mu^{3}$ in this work). Its mass is computed based on the density of the nickel $\left(8908 \mathrm{~kg} / \mathrm{m}^{3}\right)$.

The drag coefficient and the magnetization of the particle have been evaluated for particles of $100 \mu \mathrm{m}$ from experimental data obtained in open loop. The methodology used is detailed in [18]. The numerical value of the drag coefficient is: $k=3.3910-7 \mathrm{~kg} / \mathrm{s}$. The magnetization of the particle for a magnetic field of $20 \mathrm{mT}$ is $1.810^{4} \mathrm{~A} / \mathrm{m}$ which corresponds to a magnetic susceptibility $\chi=0.910^{6}$ A. $m^{-1} \cdot T^{-1}$. The maximum magnetization of the particle is thus one order of magnitude less than the saturation value (around $4.710^{5} \mathrm{~A} / \mathrm{m}$ for the nickel [24]). To get these values, the system must be repeatable so that data can be extracted from the open loop experiments. As shown in [18] the open loop response of the particles used in this work is not repeatable enough to perform the identification of $k$ and $M$, so the drag coefficient and the magnetization are considered to be the same as the ones of the $100 \mu \mathrm{m}$ particles. Since the particle is controlled in closed loop an accurate dynamic model is not required, which justifies the use of the previously identified values for the particles used in this work.

In case a more accurate identification is needed, in particular if the particle needs to be controlled in open loop, a VSM (Vibrating Sample Magnetometer) can be used to get accurate measurements of the magnetization. In addition, at Stoke regime, the drag coefficient is in the order of the characteristic length $L$ of the particle. The drag coefficient of a particle of characteristic length $l$ could thus be obtained by applying a corrective term $(l / L)$ to the identified value. 


\section{Analysis of inertia for different velocities based on experimental measurements}

\subsection{D closed loop control for repeatable trajectory tracking}

As detailed in [18] precise open loop positioning of the particle is very challenging as the size of the particle decreases and becomes smaller than $100 \mu \mathrm{m}$. So that repeatable trajectories are obtained for particles as small as $60 \mu \mathrm{m}$ closed loop control is thus proposed in this article. It is based on the kinetic model (Eq. (9)). In this section all the plots are obtained from experimental data.

The general principle of the controller is described in Fig. 4. The system is considered as decoupled, which enables to consider two independent controllers, for the $x$ and $y$ position respectively. The kinetic model is a non-linear model regarding the control variables $I_{x}$ and $I_{y}$. We have used a feed-forward linearisation considering two new inputs which are the estimated magnetic forces $\tilde{F}_{m x}$ and $\tilde{F}_{m y}$ defined by :

$$
\left\{\begin{array}{l}
\tilde{F}_{m x}=2 V \chi f(0) f^{\prime}(0) \operatorname{sign}\left(I_{x}\right) I_{x}^{2} \\
\tilde{F}_{m y}=2 V \chi f(0) f^{\prime}(0) \operatorname{sign}\left(I_{y}\right) I_{y}^{2}
\end{array} .\right.
$$

Two proportional integral controllers (PI) are used. The closed loop system is thus a second order system. To avoid oscillations a damping coefficient greater than one (overdamped system) is chosen. In that case the integral term should be greater than the response time of the open loop system (which is $450 \mathrm{~ms}$ ). It is set experimentally to $I=1129 \mathrm{~ms}$. The proportional term is then set experimentally. Its value is chosen to minimize the trajectory tracking error. It is set to $P=2,210^{-4} \mathrm{Nm}^{-1}$.



Fig. 4 Block diagram of the closed loop control performed on the experimental setup. 


\subsection{Experimental conditions}

The aim of this paper is to highlight the influence of inertia of micrometer size objects. The smallest the objects are, the most relevant will be the conclusions. However it is necessary to get repeatable trajectory tracking to perform a correct analysis. A good trade off is given by a $60 \times 50 \times 25 \mu^{3}$ particle. So that the effect of inertia can be seen clearly, the reference trajectory is chosen so that cusp-shaped regions are present. A "heart like" reference trajectory is selected. Due to the curvature of the meniscus the initial position of the particle is approximately the center of the reservoir.

Since it is technically not possible to vary the mass of the object while keeping the same magnetic properties and drag coefficient this paper proposes to analyze the trajectory of the object for different inputs. Four experiments are performed. Velocity varies with each experiment. The particle is controlled to perform the reference trajectory in respectively $T=1 \mathrm{~s}, T=500 \mathrm{~ms}$, $T=250 \mathrm{~ms}$ and $T=100 \mathrm{~ms}$, to analyze the influence of inertia with respect to the velocity. For each experiment (i.e. for each given velocity) the reference trajectory is performed 5 times to analyze the repeatability of the system and ensure that errors are due to a physical phenomenon inherent to the system and not to random noise.

\subsection{Inertia at the micrometer scale}

Fig. 5 depicts the position of the particle during the most relevant experiments ( $T=1 \mathrm{~s}, T=250 \mathrm{~ms}$ and $T=100 \mathrm{~ms}$ ). Images of the particle during the experiment $T=1 \mathrm{~s}$ are given in Fig. 2 .

At high velocity errors appear in trajectory tracking. For a given velocity the reference trajectory is performed 5 times. The deformation of the trajectory remains the same for the different periods: the system is repeatable. The errors in the trajectory tracking are not due to noise but rather to a physical phenomenon inherent to our system.

The correspondence between the position of the particle and the currents set to the coils is analyzed in Fig. 6. In the case of the slowest trajectory ( $T=1 \mathrm{~s})$, the cusp-shaped regions highlighted by the letters $\mathrm{A}, \mathrm{B}, \mathrm{C}$ and $\mathrm{D}$ in Fig. 5 correspond to the instant when the current changes sign (from positive to negative and conversely). For example at position $\mathrm{B}, I_{x}=0$ (see Fig. 6(b)). It corresponds to the instant when the $x$ position stops decreasing and starts increasing (see Fig. 6(a)), which is the left part of the heart shape (Fig. 5(b)). At C, $I_{y}=0$ and similarly the $y$ position stops decreasing and starts increasing. It corresponds to the bottom part of the heart shape. The direction of the force is directly given by the sign of the current (see Eq. (7)). The velocity vector is thus colinear to the force vector during all the experiment.

In case of a fast trajectory $(T=100 \mathrm{~ms})$ this is no longer true. For example at $\mathrm{D}$, the current $I_{x}$ is positive and increasing (Fig. $6(\mathrm{~d})$ ) whereas the $x$ position 


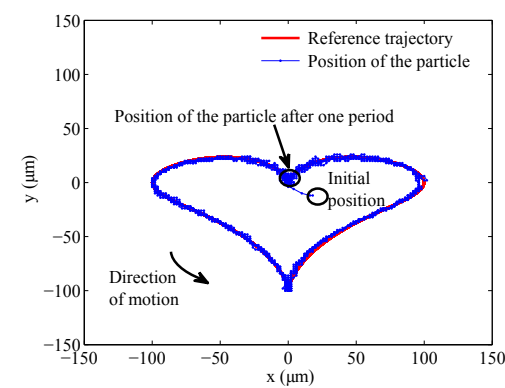

(a) $T=1 \mathrm{~s}$. Position of the particle for the first 5 periods.

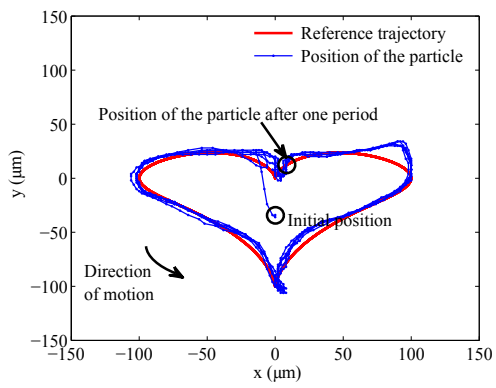

(c) $T=250 \mathrm{~ms}$. Position of the particle for the first 5 periods.

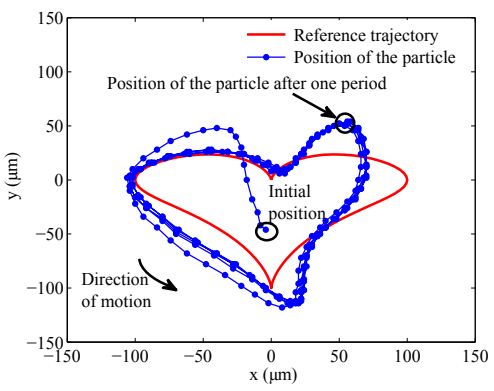

(e) $T=100 \mathrm{~ms}$. Position of the particle for the first 5 periods.

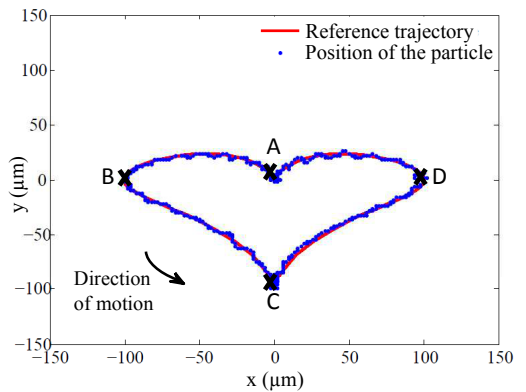

(b) $T=1 \mathrm{~s}$. Position of the particle during the second period.

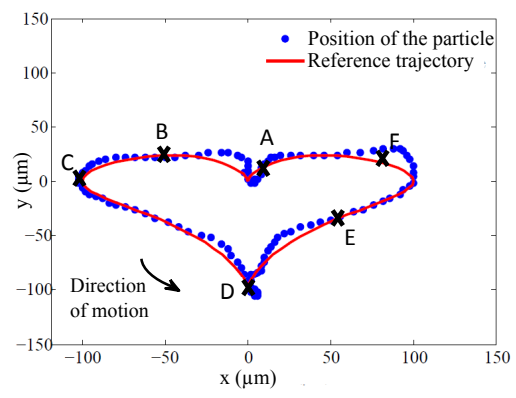

(d) $T=250 \mathrm{~ms}$. Position of the particle during the second period.

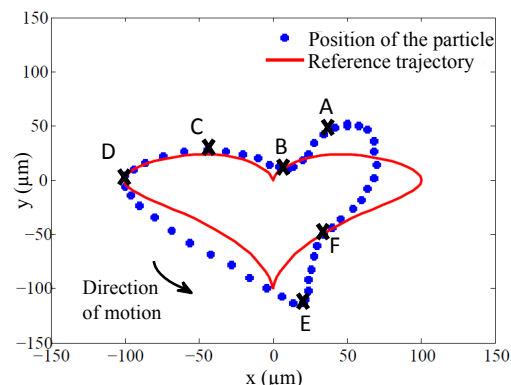

(f) $T=100 \mathrm{~ms}$. Position of the particle during the second period.

Fig. 52 D trajectory tracking of a "heart-like" shape for different velocities. A 60x50x $25 \mu^{3}$ particle is controlled to perform one complete shape (i.e. one "heart") in $T$ seconds.

stops decreasing and starts increasing (Fig. 6(c)). $I_{x}$ changed sign around $5.7 \mathrm{~ms}$ before. This phenomenon is typically due to inertia.

Other phenomena, such as the magnetic coupling between the coils or errors in the identification of the parameters of the particle, might play a role in the error of trajectory tracking. However, a phase lag appears between the 
reference and the actual position of the particle for high velocities (see Fig. 6(a) and Fig. 6(c)), which is typical from inertia.

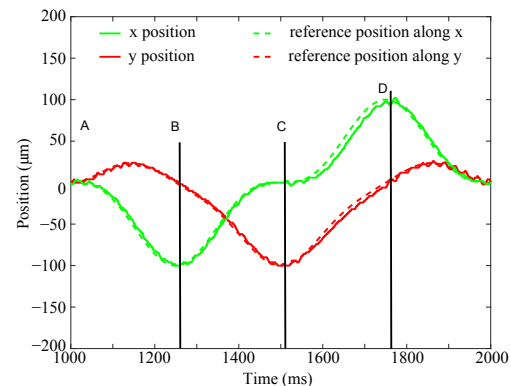

(a) $(x, y)$ position of the particle for the second period. $T=1 \mathrm{~s}$.

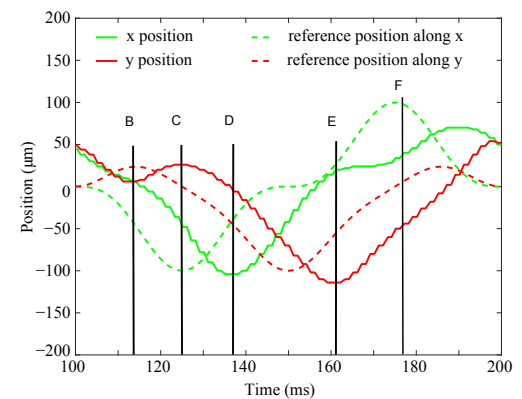

(c) $(x, y)$ position of the particle for the ( second period. $T=100 \mathrm{~ms}$.

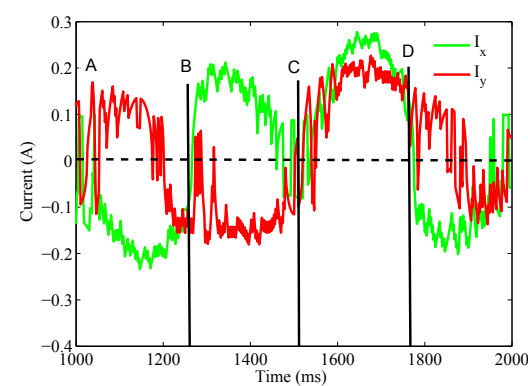

(b) $\left(I_{x}, I_{y}\right)$ currents set to the coils for the second period. $T=1 \mathrm{~s}$.

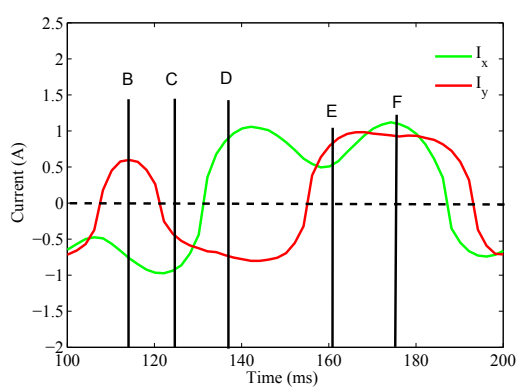

(d) $\left(I_{x}, I_{y}\right)$ currents set to the coils for the second period. $T=100 \mathrm{~ms}$.

Fig. $6(x, y)$ positions of the particle (left column) and currents set to the coils (right column) for the experiments performed in Fig. 5.

\subsection{Analysis of the performances of trajectory tracking}

The obtained trajectories are analysed in terms of velocity and precision. So that the performances can be appreciated relatively to the size of the particle the velocity is given in both $\mathrm{mm} / \mathrm{s}$, and in body length per second $(b l / \mathrm{s})$ which measures the number of time the particle moves its own size per second. The precision is given in $\mu \mathrm{m}$ and in percentage of the size of the particle. These metrics are commonly used to assess performances of micromanipulation systems [25].

Data for each plot are given in Tab. 1. For the slowest trajectories $(T=1 \mathrm{~s}$ and $T=500 \mathrm{~ms}$ ) the maximal error is three times smaller than the size of the particle. The instant velocity reaches $2.8 \mathrm{~mm} / \mathrm{s}$, which corresponds to $46.7 \mathrm{bl} / \mathrm{s}$. The common hypothesis which consists in neglecting inertia holds 
Table 1 Precision and velocity of the 2D trajectory tracking. Velocities are given in absolute value and in body length per second. Errors are given in absolute value and as a percentage of the size of the particle. $\varepsilon_{x}$ (resp. $\varepsilon_{y}$ ) is the error along the $x$ (resp. $y$ ) axis. $\varepsilon$ is the norm of the maximal error.

\begin{tabular}{|c|c|c|c|c|}
\hline$T$ & $1000 \mathrm{~ms}$ & $500 \mathrm{~ms}$ & $250 \mathrm{~ms}$ & $100 \mathrm{~ms}$ \\
\hline$\varepsilon_{x}(\mu \mathrm{m})$ & $8(13.3 \%)$ & $17(28.3 \%)$ & $42(70.0 \%)$ & $78(130.0 \%)$ \\
\hline$\varepsilon_{y}(\mu \mathrm{m})$ & $6(10.0 \%)$ & $13(21.7 \%)$ & $21(35.0 \%)$ & $57(95.0 \%)$ \\
\hline$\varepsilon(\mu \mathrm{m})$ & $10(16.7 \%)$ & $21(35.0 \%)$ & $47(78.3 \%)$ & $97(161.0 \%)$ \\
\hline \hline
\end{tabular}

\begin{tabular}{|l|c|c|c|c|c|}
\hline Instant velocity $(\mathrm{mm} / \mathrm{s})$ & $1.4(23.3 \mathrm{bl} / \mathrm{s})$ & $2.8(46.7 \mathrm{bl} / \mathrm{s})$ & $5(83.3 \mathrm{bl} / \mathrm{s})$ & $8.6(143.3 \mathrm{bl} / \mathrm{s})$ \\
\hline
\end{tabular}

Table 2 Values of the velocity and the precision error on the literature for the control of magnetically actuated micro-objects.

\begin{tabular}{|c|c|c|c|c|c|}
\hline Ref & Size $(\mu \mathrm{m})$ & DOF & Control & Precision $(\mu \mathrm{m})$ & $\begin{array}{c}\text { Velocity in mm/s } \\
\text { in body length/s })\end{array}$ \\
\hline$[20]$ & 500 & 1D & open loop &. & $556(1112)$ \\
\hline$[26]$ & $388 \times 388 \times 230$ & 1D & open loop & $\cdot$ & $133(342.8)$ \\
\hline$[25]$ & $300 \times 300 \times 70$ & $2 \mathrm{D}$ & open loop & $\cdot$ & $12.5(41.6)$ \\
\hline$[27]$ & $250 \times 130 \times 100$ & $2 \mathrm{D}$ & open loop & $\cdot$ & $10(40)$ \\
\hline$[28]$ & 42 & $2 \mathrm{D}$ & closed loop & 30 & $0.3(7.14)$ \\
\hline$[29]$ & $300 \times 60 \times 50$ & $2 \mathrm{D}$ & open loop &. & $\sim 1(2.33)$ \\
\hline$[19]$ & 50 & $2 \mathrm{D}$ & closed loop & 33 & $0.158(3.16)$ \\
\hline$[30]$ & 100 & $2 \mathrm{D}$ & closed loop & $48 \pm 59$ & $0.279(2.79)$ \\
\hline$[31]$ & $60-110$ & 2D & closed loop & 8.4 & $0.235(2.13)$ \\
\hline$[32]$ & 10 & 3D & $\cdot$ & $\cdot$ & $0.01(1)$ \\
\hline$[33]$ & $30 \times 30 \times 10$ & 2D & open loop & $\cdot$ & $0.02(0.66)$ \\
\hline$[15]$ & $100-200$ & 2D & closed loop & $4.1-40.5$ & $0.023(0.11-0.23)$ \\
\hline
\end{tabular}

at this velocity. For faster trajectories instant velocities can reach more than $8 \mathrm{~mm} / \mathrm{s}$ (140 body length per second). However deformation of the trajectory and phase lag can be seen in Fig. 5(f) and Fig. 6(c). They result in errors higher than the size of the particle (around $100 \mu \mathrm{m}$ ). Inertia should thus be taken into account in the control.

Table 2, based on [18], summarizes the performances of state of the art magnetic manipulation systems in terms of precision and velocity. The results obtained here can be compared to the data provided in that table. Work performed on particles smaller than $100 \mu \mathrm{m}$ is often limited to a few body lengths per second for which inertia can be neglected. Our closed loop control based on the kinetic model $M_{k i n}$ thus results in a good compromise between precision and velocity for $T=500 \mathrm{~ms}$. However faster trajectories still remain challenging due to inertia.

\section{Analysis of the impact of inertia}

To assess the hypothesis that the deformation of the trajectory present in the experimental plots of Sec. 3 are effectively due to inertia, numerical simulation 
is performed in this section, based on the models developed in Sec. 2. The results of simulations are then discussed and compared to conclusions derived from experiments.

\subsection{Numerical simulation with or without considering inertia}

Numerical simulation is performed using the dynamic model $M_{d y n}$ given in Sec. 2. To highlight the influence of inertia at high speed simulations based on $M_{d y n}$ are compared to simulations performed on $M_{d y n}^{w i}$ which stands for "model $M_{d y n}$ without inertia". It is obtained by neglecting the term $m \ddot{x}$ in Eq. (8). These models are used in a simulated closed loop system to replace the physical system as depicted in Fig. 7.

The simulation is performed on Matlab numerical software. The simulated trajectory corresponds to the "heart-like" shape performed experimentally. The parameters of the simulation are set to match the ones of the experimental tests. A particle of size $60 \times 50 \times 25 \mu^{3}$ is simulated. The same PI controller gains are used $\left(P=2,210^{-4} \mathrm{Nm}^{-1}\right.$ and $\left.I=1129 \mathrm{~ms}\right)$. The values of the current are computed using the kinetic model $M_{k i n}$ model. Compared to the previous section where the current was set to the coils and the position of the particle was measured by a camera, the physical system is replaced here either by the $M_{d y n}$ model to simulate a system which includes inertia or by the $M_{d y n}^{w i}$ model to simulate a system without inertia.

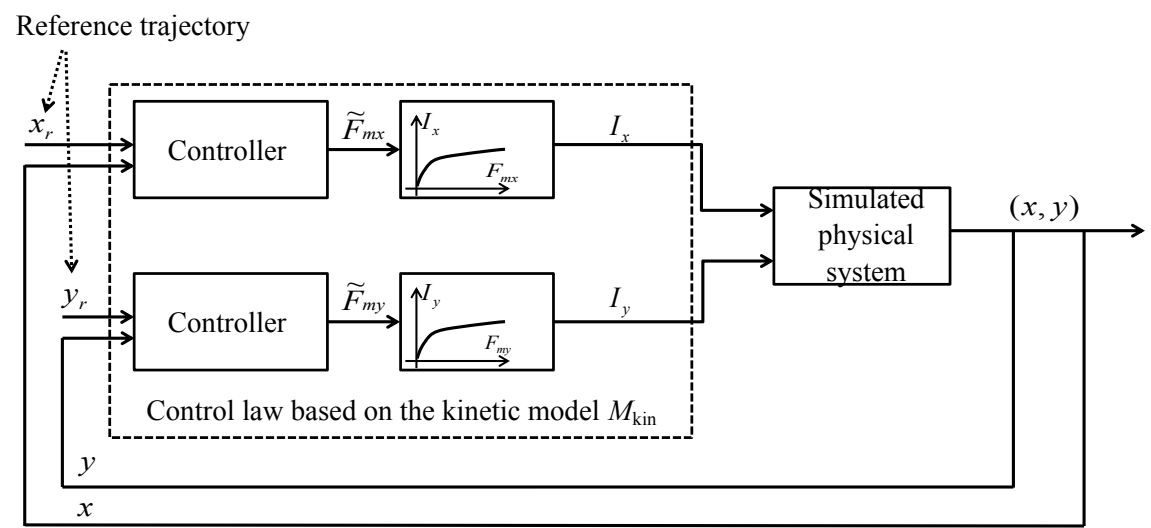

Fig. 7 Simulated closed loop control. The physical system is replaced either by the $M_{d y n}$ or by the $M_{d y n}^{w i}$ model (block "Simulated physical system").

Similarly to the experiments carried on the previous section, different velocities are considered here. Fig. 8 presents the simulated trajectories.

While using the $M_{d y n}^{w i}$ model which neglects inertia the maximum error of positioning is around $10 \mu \mathrm{m}$ for $T=100 \mathrm{~ms}$ and less than $40 \mu \mathrm{m}$ for 
$T=25 \mathrm{~ms}$. Using the $M_{d y n}$ model which takes into account the inertia of the particle, the maximum error of positioning is around $15 \mu \mathrm{m}$ for $T=100 \mathrm{~ms}$, which is similar to what is obtained using the $M_{d y n}^{w i}$ model. However, as the velocity increases the error increases and the trajectory is deformed. Errors are greater than $100 \mu \mathrm{m}$, which corresponds to $167 \%$ of the size of the particle, for $T=25 \mathrm{~ms}$. In addition the shape of the trajectory is similar between the simulated system which considers inertia and the experimental data presented in Fig. 5.

Fig. 9 presents the actual and the reference trajectories along the $x$ and $y$ axes in function of time for $T=25 \mathrm{~ms}$. A phase lag of around $4 \mathrm{~ms}$ can be seen, which is typical from inertia.

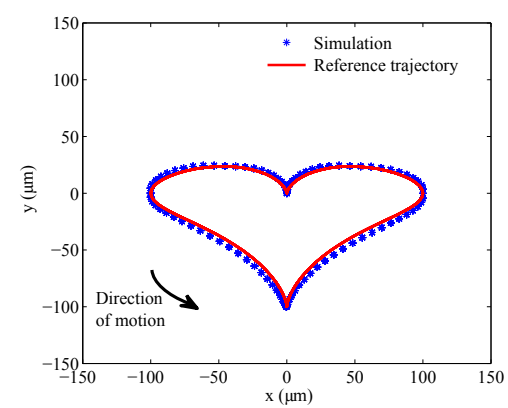

(a) Simulated trajectory using the $M_{d y n}^{w i}$ model which neglects inertia - $T=$ $100 \mathrm{~ms}$.

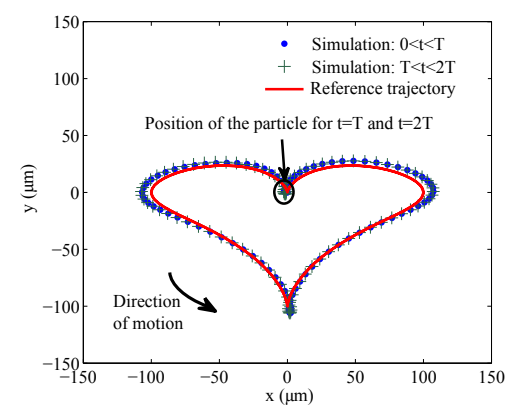

(c) Simulated trajectory using the $M_{d y n}$ model which considers inertia $-T=$ $100 \mathrm{~ms}$.

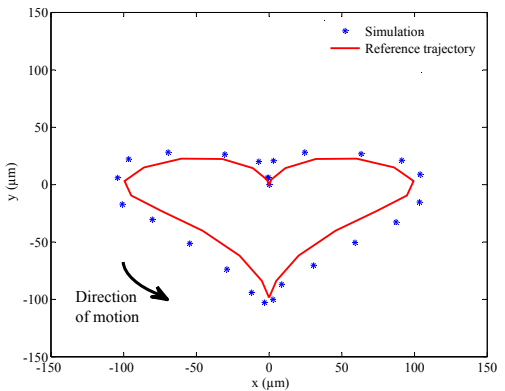

(b) Simulated trajectory using the $M_{d y n}^{w i}$ model which neglects inertia $T \stackrel{d y}{=} 25 \mathrm{~ms}$

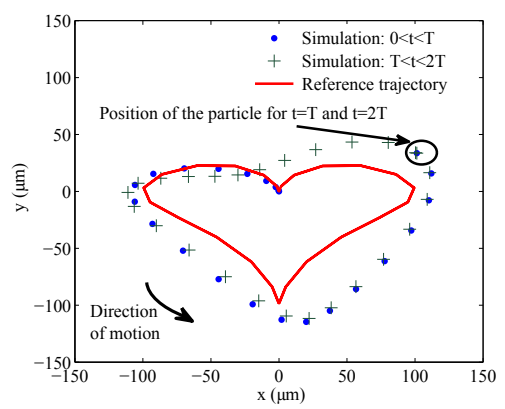

(d) Simulated trajectory using the $M_{d y n}$ model which considers inertia- $T=25 \mathrm{~ms}$

Fig. 8 Position of the particle simulated either using the $M_{d y n}^{w i}$ or the $M_{d y n}$ models which neglects or not inertia for different velocities.

This simulation corroborates the fact that the cause of the deformation of the experimental trajectory of the particle is due to the effect of inertia. Even for particles as small as $60 \mu \mathrm{m}$ it should be taken into account for the 


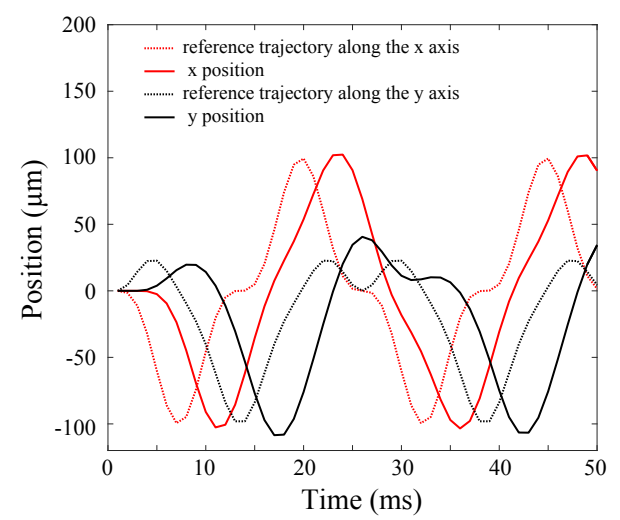

Fig. 9 Comparison between the actual and the reference position of the particle along the $x$ and $y$ axes using the $M_{d y n}$ model which considers inertia $-T=25 \mathrm{~ms}$

Table 3 Comparison between the estimated maximal magnetic force and the estimated maximal inertial term (based on data obtained from the numerical simulations) along a trajectory for different velocities.

\begin{tabular}{|c|c|c|c|c|c|}
\hline$T$ & $1000 \mathrm{~ms}$ & $250 \mathrm{~ms}$ & $100 \mathrm{~ms}$ & $50 \mathrm{~ms}$ & $25 \mathrm{~ms}$ \\
\hline Inertial term $m \ddot{x}(\mathrm{nN})$ & 0.005 & 0.025 & 0.85 & 3.7 & 9.3 \\
\hline Magnetic force $(\mathrm{nN})$ & 0.3 & 0.6 & 3.1 & 7.1 & 14 \\
\hline Inertial term/Magnetic force & 0.017 & 0.042 & 0.27 & 0.52 & 0.66 \\
\hline
\end{tabular}

control at high speed. To evaluate the impact of inertia when the velocity of the particle increases, the maximal magnetic force applied to the particle is compared to the maximal inertial term $(m \ddot{x})$. Table 3 compares these values, based on a numerical estimation of the magnetic force and the inertial term during the simulated trajectories. In case of slow trajectories $(T>100 \mathrm{~ms})$ the magnetic force is two order of magnitude higher than the inertial term. However, for faster trajectories these two terms are similar in magnitude: in case of the fastest simulated trajectory $(T=25 \mathrm{~ms})$ the inertial term is higher than half of the magnetic force. Inertia cannot be neglected anymore.

\subsection{Discussion}

As the velocity increases phase lag appears in the trajectory of the particle, for both the simulated and the experimental trajectories. Phase lag is typical from inertia. This inertia can come from three different elements: (i) the magnetic actuation system, (ii) the particle and (iii) the fluid. The time constant relative to the establishment of the magnetic field can be neglected. The numerical simulation (Fig. 8) highlights the fact that the inertia of the particle has indeed an influence on the trajectory at high velocity. This is shown by comparing the trajectory of the particle using a model which neglects inertia with respect to the trajectory obtained with a model which takes into account 
Table 4 Reynolds and Weber numbers estimated from experimental data [34].

\begin{tabular}{|c|c|c|c|c|}
\hline$T$ & $1000 \mathrm{~ms}$ & $500 \mathrm{~ms}$ & $250 \mathrm{~ms}$ & $100 \mathrm{~ms}$ \\
\hline Reynolds number & 0.07 & 0.15 & 0.26 & 0.45 \\
\hline Weber number & $1.4 .10^{-6}$ & $5.6 .10^{-6}$ & $1.8 .10^{-5}$ & $5.3 .10^{-5}$ \\
\hline
\end{tabular}

the inertia. However the phase lag and errors in trajectory tracking appear for velocities slower in real experiments $(T=100 \mathrm{~ms})$ than in simulation ( $T=25 \mathrm{~ms})$. One explanation is that the displacement of the particle induces fluid movement below the air/water interface. Inertia might be due to both the mass of the object and the one of the fluid which moves with the object. This claim is supported by the values of the Reynolds number (Table 4) computed for each experimental trajectories based on the velocities given in Table 1. For high velocities the Reynolds number, which measures the ratio of inertial forces to viscous forces within a fluid, is equal to 0.45 . It means that inertial forces become significant compared to viscous forces [34]. To corroborate this explanation additional simulation of fluid flow should be performed in future works.

The Weber number (Table 4) remains well below one. It means that fluid's inertia remains low compared to its surface tension [34]. The assumption made in Sec. 2.3 about the fact that the air/liquid interface should thus not be deformed by the particle is corroborated despite the high velocity.

Advanced control laws would undoubtedly improve trajectory tracking at high frequency. Better results are foreseen by replacing the PI controller by a predictive control law. The goal of the generalized predictive control is to find the optimal future control actions which drive the future process output to track the reference trajectory as closely as possible based on the system model in the presence of system constraints and disturbances [35]. This control law could be implemented in future work based on the model presented in Sec. 2.3.

\section{Conclusion and future work}

This article analyzes the impact of the inertia of the objects at the micrometer scale. In the literature inertia is commonly neglected. However, this article shows that even for particles as small as $60 \mu \mathrm{m}$, it is possible to reach velocities at which inertia must be taken into account to perform precise trajectory tracking.

Trajectory tracking of a complex shape which presents cusp-shaped regions and different curvature regions is performed using a $60 \mu \mathrm{m}$ particle placed at the air/liquid interface and actuated by magnetic fields. Precise positioning is obtained for velocities up to $2.8 \mathrm{~mm} / \mathrm{s}(46.7 \mathrm{bl} / \mathrm{s})$, for which inertia can be neglected. However, as the velocity increases (up to $140 \mathrm{bl} / \mathrm{s}$ are demonstrated in this paper) positioning accuracy decreases and phase lag appears, which is 
typical from inertia. Despite the small size of the particle (less than $60 \mu \mathrm{m}$ ) simulations show that its inertia must be taken into account to get precise positioning.

Increasing the speed of micromanipulation will be one of the key challenge of future micromanipulation systems. It will open the door to high speed cell sorting and high speed microassembly of artificial components. The analysis provided in this paper will be of utmost importance for these future micromanipulation systems.

Acknowledgements This work has been supported by the Labex ACTION project (contract "ANR-11-LABX-01-01"), by the ANR Multiflag project (contract "ANR-16-CE330019"), by the "Région Franche Comté" and by the French RENATECH network and its FEMTO-ST technological facility.

\section{References}

1. M. Kharboutly and M. Gauthier, "High speed closed loop control of a dielectrophoresisbased system." in IEEE International Conference on Robotics and Automation, 2013, pp. 1438-1443.

2. S. Chowdhury, W. Jing, and D. J. Cappelleri, "Controlling multiple microrobots: recent progress and future challenges," Journal of Micro-Bio Robotics, vol. 10, no. 1, pp. 1-11, 2015 .

3. J. Cheng, M. A. Rahman, and A. T. Ohta, "Optical manipulation of cells," in $M i$ crotechnology for Cell Manipulation and Sorting. Springer, 2017, pp. 93-128.

4. J. Zemanek, J. Drs, and Z. Hurak, "Dielectrophoretic actuation strategy for micromanipulation along complex trajectories," in IEEE/ASME International Conference on Advanced Intelligent Mechatronics, 2014, pp. 19-25.

5. C. Jiang and J. K. Mills, "Planar Cell Orientation Control System Using a Rotating Electric Field," IEEE/ASME Transactions on Mechatronics, vol. 20, no. 5, pp. 23502358, 2015.

6. Ultrasonic Micro/Nano Manipulations: Principles and Examples. World Scientific Publishing Company, 2014.

7. Q. Zhou, V. Sariola, K. Latifi, and V. Liimatainen, "Controlling the motion of multiple objects on a Chladni plate," Nature Communications, vol. 7, p. 12764, 2016.

8. E. B. Steager, M. S. Sakar, C. Magee, M. Kennedy, A. Cowley, and V. Kumar, "Automated biomanipulation of single cells using magnetic microrobots," The International Journal of Robotics Research, vol. 32, no. 3, pp. 346-359, 2013.

9. I. S. M. Khalil, P. Ferreira, R. Eleuterio, C. L. de Korte, and S. Misra, "Magnetic-based closed-loop control of paramagnetic microparticles using ultrasound feedback," in IEEE International Conference on Robotics and Automation, 2014, pp. 3807-3812.

10. J. Giltinan, E. Diller, and M. Sitti, "Programmable assembly of heterogeneous microparts by an untethered mobile capillary microgripper," Lab on a Chip, 2016.

11. A. Thakur, S. Chowdhury, P. Svec, C. Wang, W. Losert, and S. K. Gupta, "Indirect pushing based automated micromanipulation of biological cells using optical tweezers," The International Journal of Robotics Research, p. 0278364914523690, 2014.

12. H. Marino, C. Bergeles, and B. J. Nelson, "Robust electromagnetic control of microrobots under force and localization uncertainties," IEEE Transactions on Automation Science and Engineering, vol. 11, no. 1, pp. 310 - 316, 2014.

13. M. Sitti, H. Ceylan, W. Hu, J. Giltinan, M. Turan, S. Yim, and E. Diller, "Biomedical Applications of Untethered Mobile Milli/Microrobots," Proceedings of the IEEE, vol. 103, no. 2, pp. 205-224, 2015.

14. S. Bouchebout, A. Bolopion, M. Gauthier, and S. Régnier, "Position control of a ferromagnetic micro-particle in a dry environment," in IEEE International Conference on Advanced Intelligent Mechatronics, 2014. 
15. J. A. Piepmeier, S. Firebaugh, and C. S. Olsen, "Uncalibrated visual servo control of magnetically actuated microrobots in a fluid environment," Micromachines, vol. 5 , no. 4, pp. 797-813, 2014.

16. J. M. Ng, M. J. Fuerstman, B. A. Grzybowski, H. A. Stone, and G. M. Whitesides, "Self-assembly of gears at a fluid/air interface," Journal of the American Chemical Society, vol. 125, no. 26, pp. 7948-7958, 2003.

17. A. Jesacher, S. Fürhapter, C. Maurer, S. Bernet, and M. Ritsch-Marte, "Holographic optical tweezers for object manipulations at an air-liquid surface," Optics express, vol. 14 no. 13 , pp. 6342-6352, 2006.

18. M. Dkhil, M. Kharboutly, A. Bolopion, S. Régnier, and M. Gauthier, "Closed loop control of a magnetic particle at the air/liquid interface," IEEE Transactions on Automation Science and Engineering, pp. 1-13, jun 2015.

19. S. M. I. Khalil, Y. Kareem, S. Alonso, and M. Sarthak, "Magnetic-based motion control of sperm-shaped microrobots using weak oscillating magnetic field," in IEEE International Conference on Intelligent Robots and Systems, 2014.

20. H. Salmon, L. Couraud, and G. Hwang, "Swimming property characterizations of magnetic polarizable microrobots," in IEEE International Conference on Robotics and Automation, 2013.

21. J. Zhang, O. Onaizah, K. Middleton, L. You, and E. Diller, "Reliable grasping of threedimensional untethered mobile magnetic microgripper for autonomous pick-and-place," IEEE Robotics and Automation Letters, vol. 2, no. 2, pp. 835-840, 2017.

22. M. Dkhil, A. Bolopion, S. Régnier, and M. Gauthier, "Modeling and 1d control of a non contact magnetic actuation platform at the air/liquid interface for micrometer scale applications," in International Conference on Manipulation, Automation and Robotics at Small Scales, 2016, pp. 1-6.

23. A. Ivan, G. Hwang, J. Agnus, N. Chaillet, and S. Régnier, "Nist micrororobotics challenge. magpier: The fastest mobile microrobots in the world," IEEE Robotics and Automation Magazine, vol. 20, no. 2, pp. 63-70, 2013.

24. K. Mulyokov, G. Korznikov, R. Abdulov, and R. Valiev, "Magnetic hysteretic properties of submicron grained nickel and their variations upon annealing," Journal of magnetism and magnetic materials, vol. 89, no. 1-2, pp. 207-213, 1990.

25. D. R. Frutiger, K. Vollmers, B. E. Kratochvil, and B. J. Nelson, "Small, fast, and under control: wireless resonant magnetic micro-agents," The International Journal of Robotics Research, vol. 29, no. 5, pp. 613-636, 2010.

26. I. A. Ivan, G. Hwang, J. Agnus, M. Rakotondrabe, N. Chaillet, and S. Régnier, "First experiments on magpier: a planar wireless magnetic and piezoelectric microrobot," in IEEE International Conference on Robotics and Automation, 2011, pp. 102-108.

27. C. Pawashe, S. Floyd, and M. Sitti, "Modeling and experimental characterization of an untethered magnetic micro-robot," The International Journal of Robotics Research, vol. 28, no. 8, pp. 1077-1094, 2009.

28. A. Barbot, D. Dominique, and H. Gilgueng, "Controllable roll-to-swim motion transition of helical nanoswimmers," in IEEE International Conference on Robotics and Automation, 2014.

29. H.-W. Tung, K. E. Peyer, D. F. Sargent, and B. J. Nelson, "Noncontact manipulation using a transversely magnetized rolling robot," Applied Physics Letters, vol. 103, no. 11, p. 114101, 2013.

30. I. S. Khalil, V. Magdanz, S. Sanchez, O. G. Schmidt, and S. Misra, "The control of selfpropelled microjets inside a microchannel with time-varying flow rates," Transactions on Robotics, vol. 30, no. 1, pp. 49-58, 2014.

31. J. D. Keuning, J. de Vriesy, L. Abelmanny, and S. Misra, "Image-based magnetic control of paramagnetic microparticles in water," in IEEE International Conference on Intelligent Robots and Systems, 2011, pp. 421-426.

32. S. Schurle, K. E. Peyer, B. Kratochvil, and B. J. Nelson, "Holonomic 5-dof magnetic control of 1d nanostructures," in IEEE International Conference on Robotics and Automation, 2012, pp. 1081-1086.

33. M. S. Sakar, E. B. Steager, A. Cowley, V. Kumar, and G. J. Pappas, "Wireless manipulation of single cells using magnetic microtransporters," in IEEE International Conference on Robotics and Automation, 2011, pp. 2668-2673. 
34. H. U. Walter, Fluid sciences and materials science in space: a European perspective. Springer Science \& Business Media, 2012.

35. D. Clarke, C. Mohtadi, and P. Tuffs, "Generalized predictive control part i. the basic algorithm," Automatica, vol. 23, no. 2, pp. 137 - 148, 1987. 